\title{
Knockdown of FAM225B inhibits the progression of the hypertrophic scar following glaucoma surgery by inhibiting autophagy
}

\author{
XIANPENG MA and LILI LIU \\ Department of Dermatology, Affiliated Hospital of Beihua University, Jilin, Jilin 132001, P.R. China
}

Received August 25, 2020; Accepted December 18, 2020

DOI: $10.3892 / \mathrm{mmr} .2021 .11843$

\begin{abstract}
The formation of a hypertrophic scar (HS) may lead to failure of glaucoma surgery. Long non-coding RNAs (lncRNAs) are involved in the formation of HSs. Moreover, family with sequence similarity 225 member B (FAM225B) is upregulated in HS. However, the role of the lncRNA FAM225B in HS remains unknown. Thus, the present study aimed to investigate the function of FAM225B in HS. Scar fibroblasts were isolated from patients who had undergone glaucoma surgery. Western blotting was used to detect the expressions of Bax, Bcl-2, cleaved caspase 3, p62, ATG7 and Beclin 1, and reverse transcription-quantitative PCR (RT-qPCR) were conducted to determine the level of FAM225B in scar fibroblasts. Microtubule associated protein 1 light chain $3 \alpha$ staining was performed to examine autophagosomes in scar fibroblasts. Furthermore, cell proliferation was evaluated via 5-ethynyl-2'-deoxyuridine staining. Flow cytometry was conducted to determine cell apoptosis and the levels of reactive oxygen species (ROS) in scar fibroblasts. The cell migratory ability was assessed using a Transwell assay. The results demonstrated that FAM225B knockdown significantly attenuated scar fibroblast proliferation and induced apoptosis. Additionally, transfection of scar fibroblasts with FAM225B small interfering RNA (siRNA) significantly increased the ROS levels and significantly decreased the migration of scar fibroblasts. The FAM225B overexpression-induced increase of scar fibroblast proliferation and migration was significantly reversed by 3 -methyladenine administration. The results suggested that knockdown of FAM225B significantly inhibited the proliferation of scar fibroblasts by inhibiting autophagy. Therefore, knockdown of FAM225B could inhibit scar fibroblast proliferation after glaucoma surgery by inhibiting autophagy. These findings may provide a novel perspective of
\end{abstract}

Correspondence to: Dr Lili Liu, Department of Dermatology, Affiliated Hospital of Beihua University, 12 Jiefang Middle Road, Jilin, Jilin 132001, P.R. China

E-mail: liulili_12@126.com

Key words: scar fibroblasts, family with sequence similarity 225 member B, hypertrophic scar, autophagy developing treatment strategy for the patients with HSs after glaucoma surgery.

\section{Introduction}

Glaucoma is a chronic and progressive process resulting in an optic neuropathy with characteristic visual field deficits (1). Moreover, it affects $>2$ million individuals annually in the USA and is expected to drastically increase in the coming decades due to the aging population (2). If glaucoma is not treated in a timely manner, it can lead to blindness (3). Currently, filtering surgery is the major treatment for glaucoma, but the surgery can easily fail (4). For example, filtering bleb scarring following glaucoma filtering surgery is the primary cause of surgery failure $(5,6)$. Dense collagen fibrillary connective tissues abnormally thickened below the conjunctival epithelium of filtering blebs are often observed in cases of failed filtering surgery, accompanied by increased proliferation of fibroblasts, thus causing the loss of the aqueous drainage function $(7,8)$. At present, anti-metabolic drugs such as fluorouracil and mitomycin can inhibit the scar formation after surgery, but their anti-metabolic effects may also lead to a series of postoperative complications, such as ocular hypotension and filtering leakage $(9,10)$. Therefore, the development of safer strategies for treating hypertrophic scars (HSs) after glaucoma surgery is of great importance.

Long non-coding RNAs (lncRNAs) are a class of non-coding RNA transcripts with a length of 200 nucleotides $(11,12)$. lncRNAs are key mediators involved in the progression of multiple diseases $(13,14)$. For instance, lncRNA XIST can promote the progression of osteoarthritis (13) and lncRNA TUG1 can represses apoptosis, autophagy and inflammatory response by regulating miR-27a-3p/slit guidance ligand 2 in lipopolysaccharide-treated vascular endothelial cells (14). Furthermore, previous studies have revealed a close association between lncRNAs and HSs $(15,16)$. For example, overexpression of the lncRNA AC067945.2 could inhibit the progression of HSs by regulating the Wnt and vascular endothelial growth factor (VEGF) pathways (17). In addition, it has been reported that the lncRNA family with sequence similarity 225 member B (FAM225B) is upregulated in HSs (18). Thus, FAM225B may act as a mediator in formation of HSs. Since the role of FAM225B in HSs remains unknown, the present study aimed to investigate the function of FAM225B in HSs. 
Autophagy is a key cellular process, in which cytoplasmic organelles contribute to lysosomal degradation (19). In addition, activation of autophagy may result in the dysfunction of cellular organelles (20). Autophagy also affects cell survival by regulating the apoptotic cascade. This process is necessary for maintaining the cellular functions, which in turn protect the organisms against inflammatory injuries $(21,22)$. Recent reports have revealed that autophagy can inhibit the formation of HSs (23-25). These findings indicated that autophagy serves a crucial role during the formation of HS; however, the relationship between FAM225B and cell autophagy is yet to be elucidated. Based on these previous results, the present study aimed to investigate the association between FAM225B and cell autophagy in HSs.

The current study aimed to investigate the effect of FAM $225 \mathrm{~B}$ on the proliferation of scar fibroblasts. The findings of the present study could provide a novel approach for treating HS following glaucoma surgery.

\section{Materials and methods}

Isolation of scar fibroblasts. Scar fibroblasts were isolated from a patient (aged 63 years, female) who underwent glaucoma surgery in The Affiliated Hospital of Beihua University in August 2019, as previously described (26). The patient with glaucoma was diagnosed as previously described (27). The clinical and pathological data of patient were collected following their written informed consents. The present study was approved by the Ethics Committee of The Affiliated Hospital of Beihua University.

Cell culture. The scar fibroblasts were cultured in 90\% RPMI-1640 medium supplemented with $10 \%$ FBS, $1 \%$ penicillin and $1 \%$ streptomycin (all from Thermo Fisher Scientific, Inc.) in an incubator with $5 \% \mathrm{CO}_{2}$ at $37^{\circ} \mathrm{C}$.

Tissue collection. HS tissues $(\mathrm{n}=5)$ and normal tissues $(n=5)$ were obtained from The Affiliated Hospital of Beihua University between August 2019 and April 2020. HS and normal tissues were obtained from patients who underwent glaucoma surgery. Patients with glaucoma were diagnosed as previously described (27). Moreover, the patients who donated the HS tissues and the normal tissues were the same. The distributions of age and sex among the patients are listed in Table I. The present study was approved by the Institutional Ethical Committee of The Affiliated Hospital of Beihua University. Written informed consent was obtained from all participants.

The patient exclusion criteria were as follows: i) Individuals who suffered from other diseases and are currently under treatment; ii) pregnant and lactating women; iii) patients allergic to probiotics or have used/are using antibiotics recently; and iv) alcoholics (individuals who drank $\geq 5$ bottles of beer at a time, or for whom the alcohol content in the blood was at $\geq 0.08$ ) and smokers.

The patient inclusion criteria were as follows: Individuals diagnosed with glaucoma, according to the latest glaucoma diagnostic criteria formulated by the Ocular Fundus Disease Cooperative Group of the People's Republic of China (28), and patients who received glaucoma surgery.
Reverse transcription-quantitative PCR (RT-qPCR). Total RNA was extracted from scar fibroblasts or tissues using TRIzol ${ }^{\circledR}$ reagent (Takara Bio, Inc.) according to the manufacturer's protocol. Subsequently, cDNA was synthesized using PrimeScript RT reagent kit (Takara Bio, Inc.) according to the manufacturer's instructions. The temperature and duration of RT were: $37^{\circ} \mathrm{C}$ for $60 \mathrm{~min}$ and $85^{\circ} \mathrm{C}$ for $5 \mathrm{~min}$. RT-qPCR was performed using a $\mathrm{SYBR}^{\circledR}$ Premix Ex Taq $^{\mathrm{TM}}$ II kit (Takara Bio, Inc.) on a 7900HT system (Applied Biosystems; Thermo Fisher Scientific, Inc.) in triplicate under the following conditions: Initial denaturation for $10 \mathrm{~min}$ at $95^{\circ} \mathrm{C} ; 40$ cycles of $95^{\circ} \mathrm{C}$ for $15 \mathrm{sec}$ and $60^{\circ} \mathrm{C}$ for $30 \mathrm{sec}$; and final extension for $1 \mathrm{~min}$ at $60^{\circ} \mathrm{C}$. The primer sequences (Shanghai GenePharma Co., Ltd.) used were as follows: FAM225B forward, 5'-CCC TTGGATGCTTGGAGTGA-3' and reverse, 5'-GCGACGGTG CTGAATCTTGT-3'; p62 forward, 5'-GAACAGATGGAG TCGGATAACTG-3' and reverse, 5'-CTGGGAGAGGGACTC AATCAG-3'; Beclin 1 forward, 5'-GGCACAATCAATAAC TTCAGGC-3' and reverse, 5'-GGCAGCTCCTTAGATTTG TCTG-3'; for microtubule associated protein 1 light chain $3 \alpha$ (LC3) forward, 5'-ACCGCTGTAAGGAGGTACAGC-3' and reverse, 5'-GAAGCCGTCCTCGTCTTTCT-3'; autophagy related (ATG)7 forward, 5'-TTCCTCCTCTTGACATTTGCA G-3' and reverse, 5'-TATCTTCGTCCTTTGACCTTGG-3'; and $\beta$-actin forward, 5'-GTCCACCGCAAATGCTTCTA-3' and reverse, 5'-TGCTGTCACCTTCACCGTTC-3'. The relative expression was quantified using the $2^{-\Delta \Delta \mathrm{Cq}}$ method (29). The internal reference gene $\beta$-actin was utilized for normalization.

Cell transfection. The small interfering RNAs (siRNAs) against FAM225B (FAM225B siRNA1, FAM225B siRNA2 and FAM225B siRNA3; $10 \mathrm{nM}$ ) and negative control siRNA (siRNA-NC) were purchased from Shanghai GenePharma Co., Ltd., and were transfected into scar fibroblasts using Lipofectamine ${ }^{\circledR} 2000$ reagent (Thermo Fisher Scientific, Inc.) at $37^{\circ} \mathrm{C}$ for $48 \mathrm{~h}$ according to the manufacturer's instructions. Cells were incubated at $37^{\circ} \mathrm{C}$ for $6 \mathrm{~h}$ and the transfection efficiency was determined using RT-qPCR. The siRNA sequences were as follows: NC siRNA, 5'-GTC GCAGTACGCATACCTT-3'; FAM225B siRNA1, 5'-GCA TTGGCCTTGACCACAT-3'; FAM225B siRNA2, 5'-GCG TATGCAGACGTTGCTT-3'; and FAM225B siRNA3, 5'-GCTGCTAAGTGGCAGGTAA-3'.

For FAM225B overexpression, scar fibroblasts were transfected with pcDNA3.1 vector (NC, $10 \mathrm{nM})$ or pcDNA3.1FAM225B overexpression plasmid (FAM225B-OE, $10 \mathrm{nM}$ ) using Lipofectamine ${ }^{\circledR} 3000$ (Invitrogen; Thermo Fisher Scientific, Inc.) at $37^{\circ} \mathrm{C}$ for $48 \mathrm{~h}$. The pcDNA3.1 vector and FAM225B-OE plasmid were purchased from Shanghai GenePharma Co., Ltd.

Western blot analysis. Total proteins were isolated from scar fibroblasts using a RIPA buffer (Shanghai GenePharma Co., Ltd.) and were quantified with a BCA protein assay kit (Beyotime Institute of Biotechnology). Total proteins $(30 \mu \mathrm{g})$ were separated by $10 \%$ SDS-PAGE, and then transferred onto PVDF (Bio-Rad Laboratories, Inc.) membranes. Following blocking with $5 \%$ skimmed milk at room temperature for $1 \mathrm{~h}$, the membranes were incubated with primary antibodies 
Table I. Age and sex distribution among the patients.

\begin{tabular}{lcl}
\hline Patient & Age, years & \multicolumn{1}{c}{ Sex } \\
\hline 1 & 51 & Female \\
2 & 27 & Female \\
3 & 70 & Male \\
4 & 51 & Female \\
5 & 64 & Female \\
\hline
\end{tabular}

at $4^{\circ} \mathrm{C}$ overnight. Subsequently, membranes were incubated with horseradish peroxidase-labeled goat anti-rabbit secondary antibody (cat. no. ab7090; 1:5,000; Abcam) at room temperature for $1 \mathrm{~h}$. Enhanced chemiluminescence reagent (Thermo Fisher Scientific, Inc.) was used to visualize the protein bands. The membranes were scanned with an Odyssey Imaging system and analyzed using the Odyssey v2.0 software (Li-Cor Biosciences). The primary antibodies used in the current study were as follows: Anti-p62 (cat. no. ab109012; 1:1,000), anti-ATG7 (cat. no. ab52472; 1:1,000), anti-Beclin 1 (cat.no. ab207612; 1:1,000), anti-Bax (cat. no. ab32503; 1:1,000), anti-Bcl-2 (cat. no. ab182858; 1:1,000), anti-cleaved caspase 3 (cat. no. ab49822; 1:1,000) and anti- $\beta$-actin (cat. no. ab8226; 1:1,000; all from Abcam). $\beta$-actin served as an internal control.

Cell Counting Kit 8 (CCK-8) assay. Cell viability was tested by CCK- 8 kit according to the manufacturer's instructions. Briefly, scar fibroblasts were transfected with NC siRNA (10 nM), FAM225B siRNA1/siRNA2/siRNA3 (10 nM), FAM225B-OE (10 nM) or FAM225B-OE (10 nM) + 3-methyladenine (3-MA; MedChemExpress) for 0, 24, 48 and $72 \mathrm{~h}$ at $37^{\circ} \mathrm{C}$. Then, cells were treated with $10 \mu \mathrm{l} C \mathrm{CK}-8$ reagent (Beyotime Institute of Biotechnology) and incubated for an additional $2 \mathrm{~h}$ at $37^{\circ} \mathrm{C}$. The absorbance was measured at $450 \mathrm{~nm}$ using a microplate reader.

Immunofluorescence. Following transfected with siRNA-NC (10 nM), FAM225B siRNA2 (10 nM), FAM225B siRNA3 (10 nM), pcDNA3.1-NC (10 nM), FAM225B-OE (10 nM) or FAM225B-OE $(10 \mathrm{nM})+3-\mathrm{MA}$ at $37^{\circ} \mathrm{C}$ for $72 \mathrm{~h}$, scar fibroblasts were fixed with $4 \%$ paraformaldehyde at room temperature for 20 min, washed with PBS and blocked with 2\% BSA (Beyotime Institute of Biotechnology) at room temperature for $30 \mathrm{~min}$. Subsequently, cells were incubated with an EdU Assay/EdU Staining Proliferation kit (iFluor 647; cat. no. ab222421; Abcam) or anti-LC3 antibody (cat. no. ab63817; Abcam; $1: 1,000)$ at $4^{\circ} \mathrm{C}$ overnight. Subsequently, cells were incubated with a secondary antibody [cat. no. ab6728; IgG (horseradish peroxidase); Abcam; 1:5,000] at room temperature for $1 \mathrm{~h}$. The nuclei were stained with DAPI (Beyotime Institute of Biotechnology) at room temperature for $5 \mathrm{~min}$. Finally, cells were observed under a fluorescent microscope at a magnification of $\mathrm{x} 200$.

Transwell assay. For migration assays, the transfected cells (density, $3 \times 10^{4}$ cells/well) were resuspended in serum-free DMEM (100 $\mu$ l; Thermo Fisher Scientific, Inc.) and were then plated onto the upper chamber of the Transwell 24-well plates. Moreover, the lower chamber of each well was supplemented with $600 \mu \mathrm{l}$ DMEM containing $10 \%$ FBS. Following incubation for $24 \mathrm{~h}$ at $37^{\circ} \mathrm{C}$, the non-migrated cells on the upper surface of the membrane were removed. Subsequently, the migrated cells on the lower surface were fixed with $4 \%$ paraformaldehyde at room temperature for $15 \mathrm{~min}$, followed by staining with $0.5 \%$ crystal violet at room temperature for $30 \mathrm{~min}$. The number of migrated cells was counted under a light microscope at a magnification of x400.

Cell apoptosis assays. The early and late apoptosis (Annexin- $\mathrm{V}^{+} \mathrm{PI}^{-}$plus Annexin- $\mathrm{V}^{+} \mathrm{PI}^{+}$) of scar fibroblasts were measured via flow cytometry. Scar fibroblasts were seeded into 6 -well plates at a density of $1 \times 10^{6}$ cells/well. The residue was resuspended in $100 \mu \mathrm{l}$ binding buffer following centrifugation $\left(4^{\circ} \mathrm{C}\right)$ at $500 \mathrm{x} \mathrm{g}$ for $5 \mathrm{~min}$. Subsequently, $5 \mu \mathrm{l}$ Annexin V-FITC (BD Biosciences) and $5 \mu 1$ PI (BD Biosciences) at room temperature were added into the cell suspension for $15 \mathrm{~min}$. The cell apoptosis rate was measured using a flow cytometer (BD Biosciences) and the results were analyzed using FACS (BD Biosciences) with FlowJo (v10.6.2; FlowJo LLC).

Reactive oxygen species (ROS) detection. ROS detection was performed using a ROS detection kit (green fluorescence; Beyotime Institute of Biotechnology). Cell suspensions were collected and stained with the ROS probe 2,7-dichlorofluorescein diacetate (Beyotime Institute of Biotechnology) at room temperature for $15 \mathrm{~min}$ as previously described (30). After $20 \mathrm{~min}$ of incubation, cells were centrifuged at $300 \mathrm{x} \mathrm{g}$ $\left(4^{\circ} \mathrm{C}\right.$ for $\left.10 \mathrm{~min}\right)$, washed with PBS and resuspended. Finally, the relative ROS level was measured via flow cytometry (FACSAria II; BD Biosciences), and the data were analyzed using FlowJo 10.0 (BD Biosciences).

ELISA. The levels of superoxide dismutase (SOD) and glutathione (GSH) in the supernatants of scar fibroblasts (collected by centrifugation at $4^{\circ} \mathrm{C}$ for $10 \mathrm{~min}$ at $300 \mathrm{x} \mathrm{g}$ ) were detected using SOD ELISA kit [cat. no. CSB-EL022399; Multisciences (Lianke) Biotech Co., Ltd.] and GSH ELISA kit [cat. no. xy-E10818; Multisciences (Lianke) Biotech Co., Ltd.] according to the manufacturer's instructions.

Statistical analysis. Data are presented as the mean \pm SD. The CCK-8 assay was performed in quintuplicate. Cell transfections, RT-qPCR, immunofluorescence staining, flow cytometry, western blot analysis and the Transwell migration assay were repeated three times. GraphPad Prism software (version 7.0; GraphPad Software, Inc.) was used to analyze the data. One-way ANOVA and Tukey's tests were conducted to evaluate the differences for multiple comparisons. $\mathrm{P}<0.05$ was considered to indicate a statistically significant difference.

\section{Results}

FAM225B knockdown significantly inhibits the proliferation of scar fibroblasts. The morphology of scar fibroblasts isolated from a patient was observed under a microscope, and fibrotic scarring was observed (Fig. 1A). This morphology was consistent with the characteristics of scar fibroblasts (31). Moreover, 
A

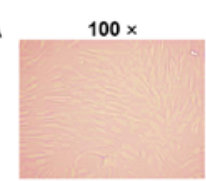

$200 \times$

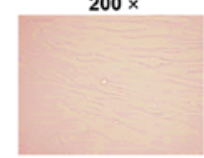

D

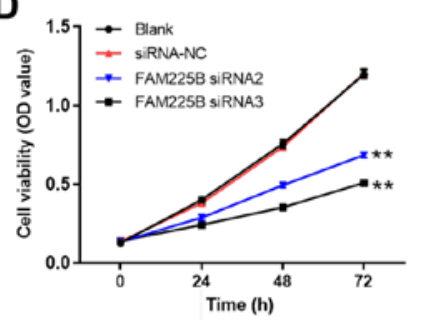

B

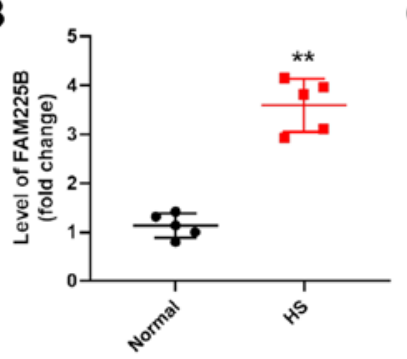

C

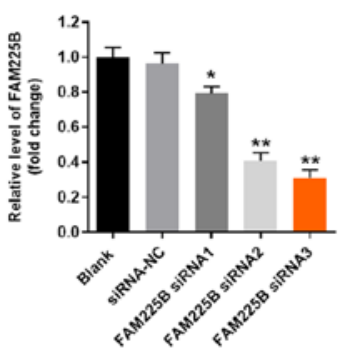

E

E Blank
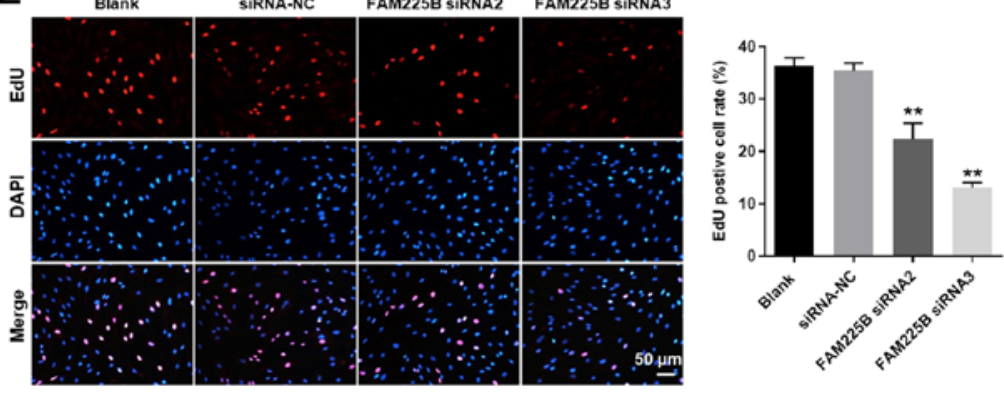

Figure 1. FAM225B knockdown significantly inhibits the proliferation of scar fibroblasts. (A) Scar fibroblast morphology was observed under a microscope. (B) Expression of FAM225B in HS or normal tissues was detected via RT-qPCR. ${ }^{* *} \mathrm{P}<0.01$ vs. normal. (C) Scar fibroblasts were transfected with FAM225B siRNA1, siRNA2 or siRNA3 for $24 \mathrm{~h}$. Then, the efficiency of cell transfection was detected via RT-qPCR. (D) Scar fibroblasts were transfected with FAM225B siRNA2 or siRNA3 for $0,24,48$ or $72 \mathrm{~h}$. Then, cell viability was assessed using a Cell Counting Kit- 8 assay. (E) Proliferation of scar fibroblasts was detected using EdU staining (magnification, x200). Red fluorescence indicates EdU, while blue fluorescence indicates DAPI. " $\mathrm{P}<0.05$, ${ }^{* *} \mathrm{P}<0.01$ vs. Blank. RT-qPCR, reverse transcription-quantitative PCR; EdU, 5-Ethynyl-2'-deoxyuridine; NC, negative control; siRNA, small interfering RNA; FAM225B, family with sequence similarity 225 member B; HS, hypertrophic scar; OD, optical density.
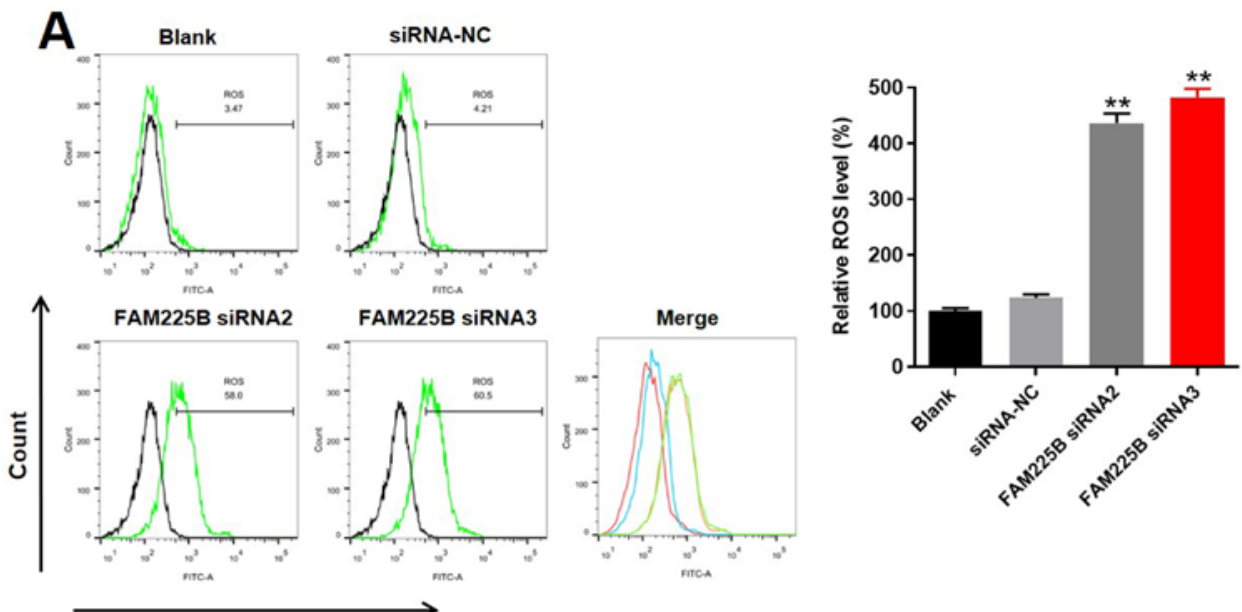

FITC-A
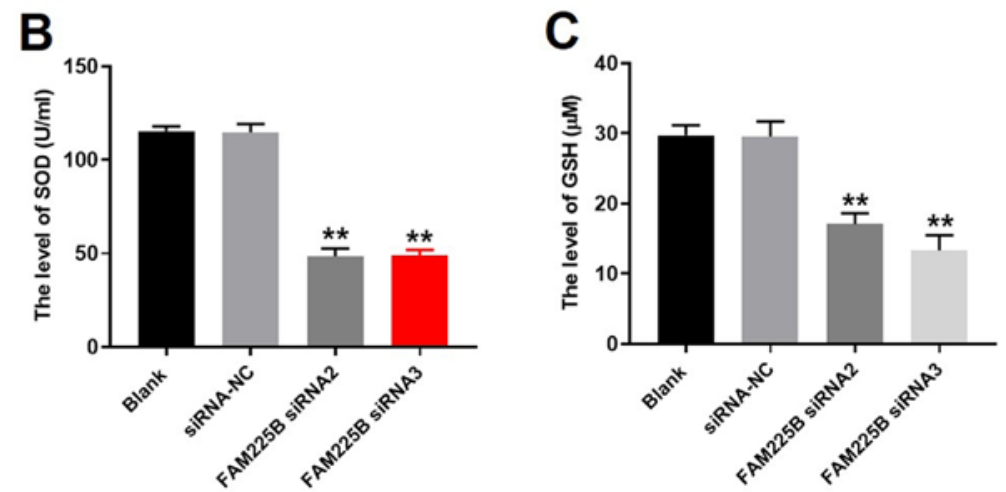

Figure 2. Knockdown of FAM225B significantly induces injury in scar fibroblasts. (A) FACS was performed to detect ROS levels in scar fibroblasts. The relative level of ROS was calculated. Levels of (B) SOD and (C) GSH in supernatants of scar fibroblasts were detected via ELISA. * P $<0.01$ vs. Blank. NC, negative control; siRNA, small interfering RNA; FAM225B, family with sequence similarity 225 member B; SOD, superoxide dismutase; GSH, glutathione; ROS, reactive oxygen species. 

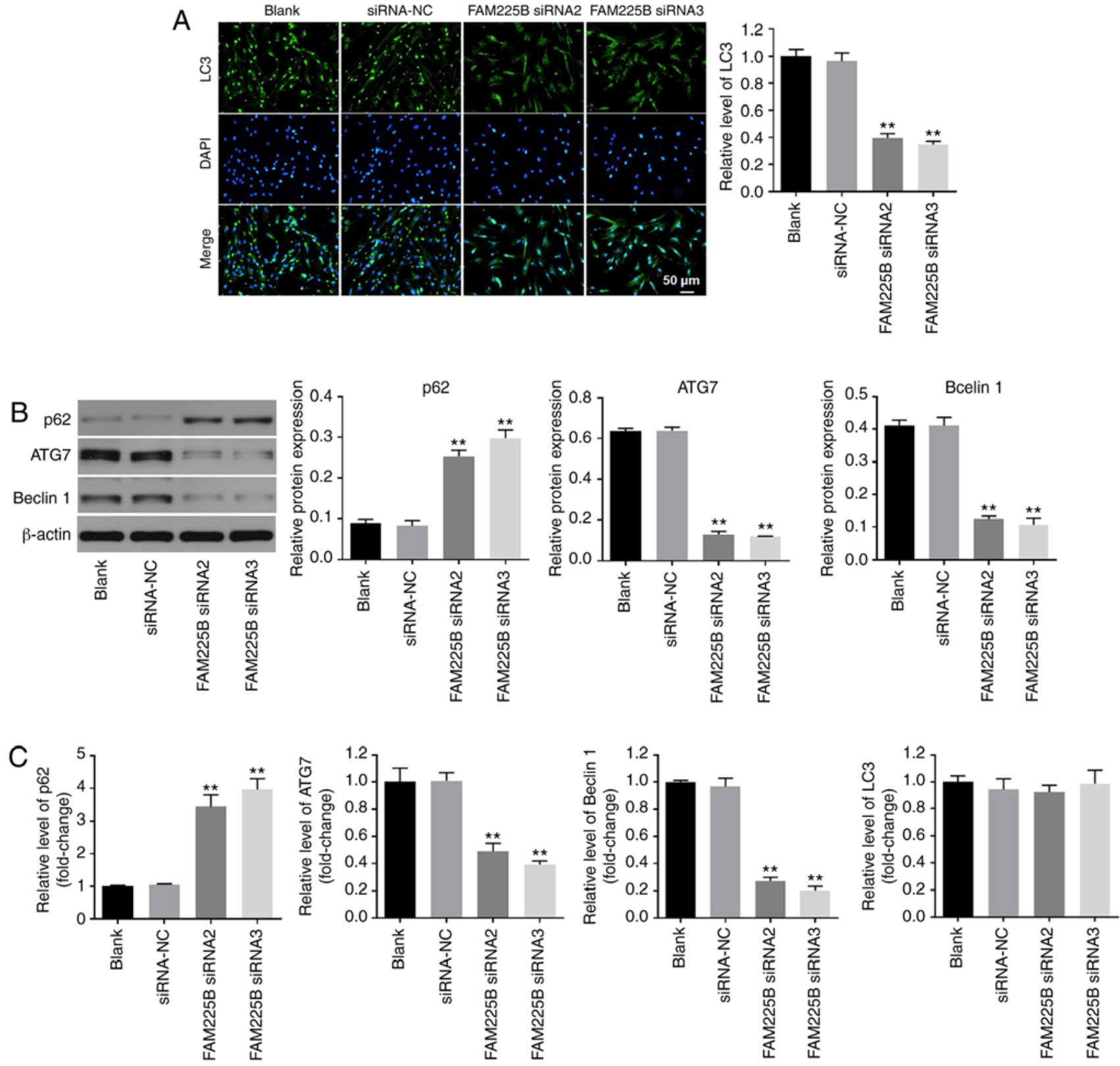

Figure 3. FAM225B siRNA inhibits the progression of hypertrophic scar in vitro by inhibiting autophagy. (A) Expression of LC3 in scar fibroblasts was detected using immunofluorescence staining (magnification, x200). The relative level of LC3 was calculated. Green fluorescence indicates LC3 and blue fluorescence indicates DAPI. (B) Protein expression levels of ATG7, Beclin 1 and p62 in scar fibroblasts were detected via western blotting. The relative protein expressions were semi-quantified by normalizing to $\beta$-actin. (C) mRNA expression levels of ATG7, Beclin 1, p62 and LC3 in scar fibroblasts were detected using reverse transcription-quantitative PCR. ${ }^{* *} \mathrm{P}<0.01$ vs. Blank. NC, negative control; siRNA, small interfering RNA; FAM225B, family with sequence similarity 225 member B; LC3, microtubule associated protein 1 light chain $3 \alpha$; ATG7, autophagy related 7.

the expression of FAM225B was significantly upregulated in HS tissues, compared with normal tissues (Fig. 1B). It was also found that the expression of FAM225B in scar fibroblasts was significantly downregulated following transfection with FAM225B siRNAs (Fig. 1C). In addition, FAM225B was more efficiently downregulated when scar fibroblasts were treated with FAM225B siRNA2 or siRNA3 compared with FAM225B siRNA1. Therefore, FAM225B siRNA2 and siRNA3 were selected to knockdown FAM225B expression in the subsequent experiments. The viability of scar fibroblasts was significantly decreased following transfection with FAM225B siRNA2 or siRNA3 (Fig. 1D). Consistently, knockdown of FAM225B significantly attenuated the proliferation of scar fibroblasts (Fig. 1E). Overall, these findings indicated that FAM225B knockdown significantly attenuated the proliferation of scar fibroblasts.

Knockdown of FAM225B notably induces oxidative stress in scar fibroblasts. To evaluate the effect of FAM225B on the levels of ROS in scar fibroblasts, flow cytometric analysis was conducted. As expected, knockdown of FAM225B significantly increased ROS levels in scar fibroblasts (Fig. 2A). In addition, the levels of SOD and GSH in the supernatants of scar fibroblasts were significantly decreased by FAM225B siRNA (Fig. 2B and C). Since the levels of ROS, SOD and GSH are closely associated with cell injury (32), the aforementioned 

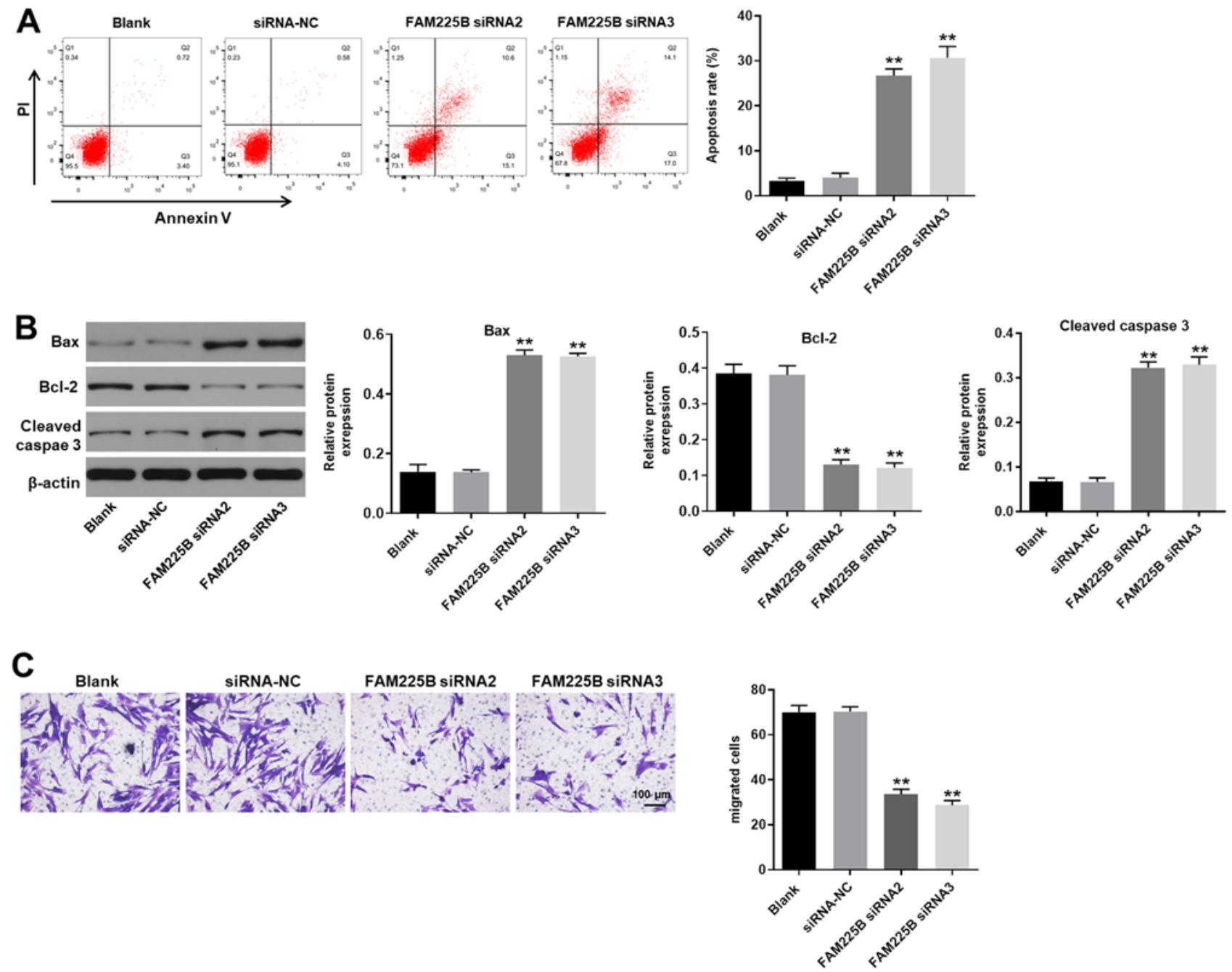

Figure 4. Knockdown of FAM225B significantly induces apoptosis and inhibits migration of scar fibroblasts. (A) Apoptosis of scar fibroblasts was detected via flow cytometry and the apoptotic rate was calculated. (B) Protein expression levels of Bax, Bcl-2 and cleaved caspase 3 in scar fibroblasts were examined via western blotting. The relative protein expression levels were semi-quantified by normalizing to $\beta$-actin. (C) Cell migration was measured using a Transwell assay (magnification, x400). ${ }^{* *} \mathrm{P}<0.01$ vs. Blank. NC, negative control; siRNA, small interfering RNA; FAM225B, family with sequence similarity 225 member B.

data suggested that knockdown of FAM225B could notably induce oxidative stress in scar fibroblasts.

FAM225B siRNA attenuates the progression of $H S$ in vitro by inhibiting autophagy. In order to evaluate the association between FAM225B and cell autophagy, immunofluorescence staining against LC3 (autophagy indicator) was performed. As presented in Fig. 3A, the expression of LC3 in scar fibroblasts was significantly decreased following FAM225B knockdown. In addition, knockdown of FAM225B significantly upregulated p62 expression (Fig. 3B and C). By contrast, the protein expression levels of ATG7 and Beclin-1 in scar fibroblasts were significantly downregulated in cells transfected with FAM225B siRNA (Fig. 3B and C). However, knockdown of FAM225B had a very limited effect on the mRNA expression of LC3 (Fig. 3C). Since LC3, p62, ATG7 and Beclin-1 are involved in the process of cell autophagy (33-35), these factors can be considered as autophagy indicators. Thus, the results indicated that FAM225B knockdown attenuated the progression of HS in vitro by inhibiting autophagy.
Knockdown of FAM225B significantly induces apoptosis and inhibits migration of scar fibroblasts. To evaluate cell apoptosis, flow cytometric analysis (Annexin V and PI staining) was performed. As presented in Fig. 4A, knockdown of FAM225B significantly induced scar fibroblast apoptosis. Furthermore, FAM225B siRNA significantly increased the expression levels of Bax and cleaved caspase 3, and downregulated those of Bcl-2 in scar fibroblasts (Fig. 4B). FAM225B knockdown also significantly inhibited the migratory ability of scar fibroblasts (Fig. 4C). Therefore, knockdown of FAM225B markedly induced apoptosis and attenuated the migration of scar fibroblasts.

Overexpression of FAM $225 B$ significantly promotes the proliferation of scar fibroblasts by inhibiting autophagy. In order to further confirm the association between FAM225B and cell autophagy in scar fibroblasts, cells were overexpressed with FAM225B, and then the efficiency of overexpression was detected. As expected, pcDNA3.1-FAM225B significantly increased the expression levels of FAM225B in scar fibroblasts (Fig. 5A). In addition, the viability of scar fibroblasts 

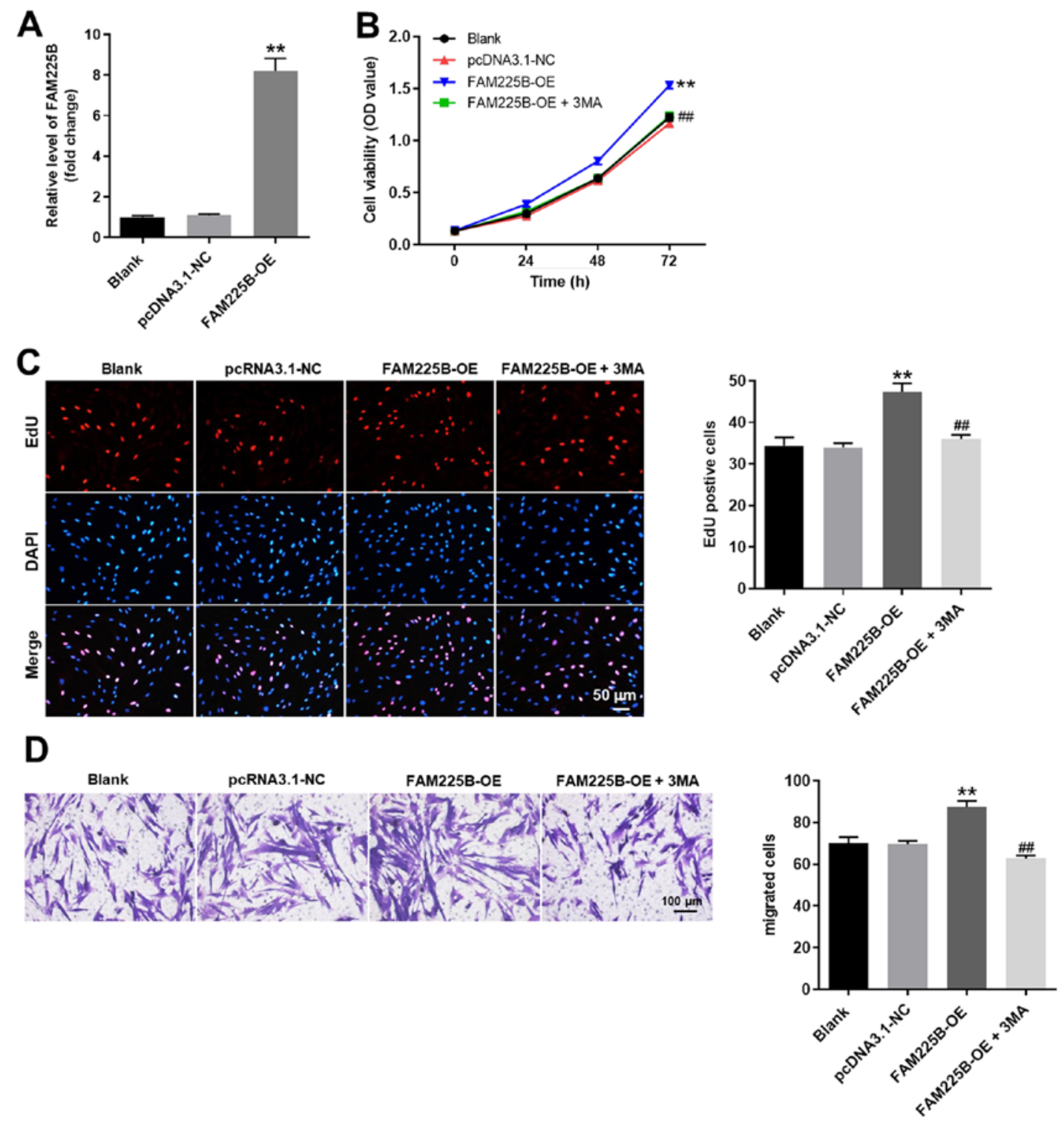

Figure 5. OE of FAM225B significantly promotes the proliferation of scar fibroblasts by inhibiting autophagy. (A) Scar fibroblasts were transfected with pcDNA3.1-FAM225B (FAM225B OE). Then, the efficiency of overexpression was detected via reverse transcription-quantitative PCR. (B) Scar fibroblasts were transfected with FAM225B OE or FAM225B OE + 3-MA. Then, cell viability was assessed using a Cell Counting Kit-8 assay. (C) Proliferation of scar fibroblasts was detected using EdU staining (magnification, $\mathrm{x} 200$ ). (D) Cell migration was detected with a Transwell assay (magnification, $\mathrm{x} 400) .{ }^{* *} \mathrm{P}<0.01 \mathrm{vs}$. Blank; "\# P<0.01 vs. FAM225B OE. NC, negative control; FAM225B, family with sequence similarity 225 member B; OE, overexpression; OD, optical density; EdU, 5-Ethynyl-2'-deoxyuridine; 3-MA, 3-methyladenine.

was significantly enhanced by FAM225B overexpression, which was partially reversed following treatment of fibroblasts with the autophagy inhibitor 3-MA (Fig. 5B). Consistently, the FAM225B overexpression-mediated increase of scar fibroblast proliferation was significantly inhibited by 3-MA (Fig. 5C). Furthermore, overexpression of FAM225B significantly enhanced the migratory ability of scar fibroblasts, which was notably reversed by 3-MA (Fig. 5D). Collectively, these results suggested that overexpression of FAM225B significantly promoted the proliferation of scar fibroblasts by inhibiting autophagy.

\section{Discussion}

Understanding the pathogenesis of HS may benefit the selection of the appropriate HS treatment strategy. Several studies have reported that non-coding RNAs, including lncRNAs, serve a key role in HS $(15,18,36)$. For example, Tu et al $(18)$ revealed that FAM225B was upregulated in HS. The results of the present study further supplemented the current knowledge regarding the effect of FAM225B on the formation of HSs, suggesting that FAM225B could serve an important role in HS progression.

Previous studies have revealed that autophagy serves key roles in the pathogenesis of HSs as it can promote the formation of HS fibroblasts $(23,37)$. For instance, activation of autophagy can inhibit proliferation of scar fibroblasts by inducing cell apoptosis (38), and autophagy can inhibit the proliferation of scar fibroblasts via the inactivation of the PI3K/Akt/mTOR pathway (39). On the contrary, the present data suggested that inhibition of autophagy could decrease the proliferation of scar fibroblasts. This discrepancy may be due to the different degrees of cell injury and oxidative stress. 
Beclin-1 is an important autophagy mediator, which increases autophagy (40). Furthermore, it has been reported that ATG7 promotes autophagy (41), while p62 is negatively associated with the autophagy process (42). The results of the current study were consistent with the aforementioned findings, suggesting that knockdown of FAM225B could inhibit the progression of HS by inhibiting autophagy.

Apoptosis is closely associated with autophagy in types of cancer, including hepatocellular carcinoma $(43,44)$. Importantly, autophagy is necessary for the onset of apoptosis, which usually triggers the occurrence of cell apoptosis and injury $(45,46)$. Based on these aforementioned findings, it can be concluded that knockdown of FAM225B induced cell injury and increased the oxidative stress of scar fibroblasts by inhibiting cell autophagy and inducing apoptosis. Moreover, a previous study reported that Proscillaridin A could induce apoptosis and autophagy in liver cancer cells (43). Thus, the association between apoptosis and autophagy requires further investigation.

There are some limitations to the present research. Firstly, this study focused only on the autophagy process. In addition, the mechanism underlying the effect of FAM225B knockdown on promoting cell autophagy remains unknown, and the microRNAs sponged by FAM225B should be evaluated. Therefore, further studies are required.

In conclusion, the present study demonstrated that knockdown of FAM225B significantly attenuating the progression of HS following glaucoma surgery by inhibiting autophagy. Therefore, the current finding may provide a new perspective of developing treatment strategy for the patients with HS after glaucoma surgery.

\section{Acknowledgements}

Not applicable.

\section{Funding}

No funding was received.

\section{Availability of data and materials}

All data generated or analyzed during this study are included in this published article.

\section{Authors' contributions}

$\mathrm{XM}$ and LL conceived and supervised the study, designed experiments and reviewed the results. Both authors read and approved the final version of the manuscript.

\section{Ethics approval and consent to participate}

The present study was approved by the Institutional Ethical Committee of The Affiliated Hospital of Beihua University. Written informed consent was obtained from all participants.

\section{Patient consent for publication}

Not applicable.

\section{Competing interests}

These authors declared that they have no competing interests.

\section{References}

1. Li X, Leng Y, Jiang Q, Wang Z, Luo P, Zhang C, Chen L, Wang Y, Wang H, Yue X, et al: Eye drops of metformin prevents fibrosis after glaucoma filtration surgery in rats via activating AMPK/Nrf2 signaling pathway. Front Pharmacol 11: 1038, 2020.

2. Swogger J, Conner IP, Rosano M, Kemmerer M, Happ-Smith C, Wells A, Schuman JS and Yates CC: Injected versus sponge-applied mitomycin C (MMC) during modified trabeculectomy in New Zealand white rabbit model. Transl Vis Sci Technol 9: 23, 2020

3. Yaman D, Takmaz T, Yüksel N, Dincer SA and Şahin FI: Evaluation of silent information regulator T (SIRT) 1 and Forkhead Box O (FOXO) transcription factor 1 and 3a genes in glaucoma. Mol Biol Rep 47: 9337-9344, 2020.

4. Cheng WS, Chen CL, Chen JT, Lin LT, Pao SI, Chen YH and Lu DW: AR12286 alleviates TGF- $\beta$-related myofibroblast transdifferentiation and reduces fibrosis after glaucoma filtration surgery. Molecules 25: 4422, 2020.

5. Yamamoto K, Kokubun T, Sato K, Akaishi T, Shimazaki A, Nakamura M, Shiga Y, Tsuda S, Omodaka K, Saya H and Nakazawa T: The DNA topoisomerase II inhibitor amsacrine as a novel candidate adjuvant in a model of glaucoma filtration surgery. Sci Rep 9: 19288, 2019.

6. Yu J, Qiu LX, Qing GP, Zhao BW and Wang H: Modified cortex mori capsules improving the successful rate of functional filtering blebs after reclinical glaucoma filtering surgery. World J Clin Cases 7: 3436-3445, 2019.

7. Zhang F, Liu K, Pan Z, Cao M, Zhou D, Liu H, Huang Y and Duan X: Effects of rosiglitazone/PHBV drug delivery system on postoperative fibrosis in rabbit glaucoma filtration surgery model. Drug Deliv 26: 812-819, 2019.

8. Sun J, Liu X, Lei Y, Tang M, Dai Z, Yang X, Yu X, Yu L, Sun X and Ding J: Sustained subconjunctival delivery of cyclosporine A using thermogelling polymers for glaucoma filtration surgery. J Mater Chem B 5: 6400-6411, 2017.

9. Holló G: Wound Healing and Glaucoma Surgery: Modulating the scarring process with conventional antimetabolites and new molecules. Dev Ophthalmol 59: 80-89, 2017.

10. Sharma A, Anumanthan G, Reyes M, Chen H, Brubaker JW, Siddiqui S, Gupta S, Rieger FG and Mohan RR: Epigenetic modification prevents excessive wound healing and scar formation after glaucoma filtration surgery. Invest Ophthalmol Vis Sci 57: 3381-3389, 2016.

11. Zhang H, Li J, Shao W and Shen N: lncRNA SNHG9 is downregulated in osteoarthritis and inhibits chondrocyte apoptosis by downregulating miR-34a through methylation. BMC Musculoskelet Disord 21: 511, 2020.

12. Wang JH, Wu XJ, Duan YZ and Li F: Circular RNA_CNST promotes the tumorigenesis of osteosarcoma cells by sponging miR-421. Cell Transplant 29: 963689720926147, 2020.

13. Xu YT, Leng YR, Liu MM, Dong RF, Bian J, Yuan LL, Zhang JG, Xia YZ and Kong LY: MicroRNA and long noncoding RNA involvement in gout and prospects for treatment. Int Immunopharmacol 87: 106842, 2020.

14. Dong Y, Fan G, Li Y and Zhou Q: TUG1 represses apoptosis, autophagy, and inflammatory response by regulating miR-27a-3p/SLIT2 in LPS-treated vascular endothelial cells. J Surg Res 256: 345-354, 2020.

15. Li M, Wang J, Liu D and Huang H: High-throughput sequencing reveals differentially expressed lncRNAs and circRNAs, and their associated functional network, in human hypertrophic scars. Mol Med Rep 18: 5669-5682, 2018.

16. Chen J, Zhou R, Liang Y, Fu X, Wang D and Wang C: Blockade of IncRNA-ASLNCS5088-enriched exosome generation in M2 macrophages by GW4869 dampens the effect of M2 macrophages on orchestrating fibroblast activation. FASEB J 33: 12200-12212, 2019.

17. Chen L, Li J, Li Q, Li X, Gao Y, Hua X, Zhou B and Li J: Overexpression of lncRNA AC067945.2 down-regulates collagen expression in skin fibroblasts and possibly correlates with the VEGF and Wnt signalling pathways. Cell Physiol Biochem 45: 761-771, 2018. 
18. Tu L, Huang Q, Fu S and Liu D: Aberrantly expressed long noncoding RNAs in hypertrophic scar fibroblasts in vitro: A microarray study. Int J Mol Med 41: 1917-1930, 2018.

19. Yamoto M, Alganabi M, Chusilp S, Lee D, Yazaki Y, Lee C, Li B and Pierro A: Lysosomal overloading and necrotizing enterocolitis. Pediatr Surg Int 36: 1157-1165, 2020.

20. Li Y, Zhang J, Zhou K, Xie L, Xiang G, Fang M, Han W, Wang X and Xiao J: Elevating sestrin2 attenuates endoplasmic reticulum stress and improves functional recovery through autophagy activation after spinal cord injury. Cell Biol Toxicol: Aug 1, 2020 (Epub ahead of print). doi: 10.1007/s10565-020-09550-4

21. Gutjahr A, Papagno L, Vernejoul F, Lioux T, Jospin F, Chanut B, Perouzel E, Rochereau N, Appay V, Verrier B and Paul S: New chimeric TLR7/NOD2 agonist is a potent adjuvant to induce mucosal immune responses. EBioMedicine 58: 102922, 2020.

22. Fu H, Chung KR, Liu X and Li H: Aaprb1, a subtilsin-like protease, required for autophagy and virulence of the tangerine pathotype of Alternaria alternata. Microbiol Res 240: 126537, 2020.

23. Shi J, Xiao H, Li J, Zhang J, Li Y, Zhang J, Wang X, Bai X, Tao K, Hu D and Guan H: Wild-type p53-modulated autophagy and autophagic fibroblast apoptosis inhibit hypertrophic scar formation. Lab Invest 98: 1423-1437, 2018.

24. Liu X, Xu Q, Long X, Liu W, Zhao Y, Hayashi T, Hattori S, Fujisaki H, Ogura T, Tashiro SI, et al: Silibinin-induced autophagy mediated by PPAR $\alpha$-sirt1-AMPK pathway participated in the regulation of type I collagen-enhanced migration in murine 3T3-L1 preadipocytes. Mol Cell Biochem 450: 1-23, 2019.

25. Kyun S, Yoo C, Park HY, Kim J and Lim K: The effects of exogenous lactate administration on the IGF1/Akt/mTOR pathway in rat skeletal muscle. Int J Environ Res Public Health 17: 7805, 2020 .

26. Wang XC, Wang T, Zhang Y, Wang LL, Zhao RY and Tan W: Tacrolimus inhibits proliferation and induces apoptosis by decreasing survivin in scar fibroblasts after glaucoma surgery. Eur Rev Med Pharmacol Sci 22: 2934-2940, 2018.

27. Jammal AA, Berchuck SI, Thompson AC, Costa VP and Medeiros FA: The effect of age on increasing susceptibility to retinal nerve fiber layer loss in glaucoma. Invest Ophthalmol Vis Sci 61: 8, 2020.

28. $\mathrm{Mu}$ Y: Correlation analysis between TCM syndromes of primary angle-closure glaucoma and type A personality. Chin J Tradit Chin Ophthalmol 10: 23-30, 2020

29. Livak KJ and Schmittgen TD: Analysis of relative gene expression data using real-time quantitative PCR and the 2(-Delta Delta C(T)) Method. Methods 25: 402-408, 2001.

30. Ye Q, Wu J, Wu P, Wang J, Niu W, Yang S, Chen M, Rehman S and Zhu N: Enhancing peroxymonosulfate activation of $\mathrm{Fe}-\mathrm{Al}$ layered double hydroxide by dissolved organic matter: Performance and mechanism. Water Res 185: 116246, 2020.

31. Wei Z, Wang Y, Jiang L, Ji N, Wang Y, Chen F, Li T, Li J, Xu H, Zeng $X$ and Chen Q: miR-223 regulates oral squamous cell carcinoma metastasis through the $\mathrm{Wnt} / \beta$-catenin signaling pathway. Oral Oncol 109: 104941, 2020.

32. Liu YP, Wan J, Long F, Tian J and Zhang C: circPVT1 facilitates invasion and metastasis by regulating $\mathrm{miR}-205-5 \mathrm{p} / \mathrm{c}-\mathrm{FLIP}$ axis in osteosarcoma. Cancer Manag Res 12: 1229-1240, 2020.

33. Zhang Y, Chang B, Zhang J and Wu X: lncRNA SOX2OT alleviates the high glucose-induced podocytes injury through autophagy induction by the miR-9/SIRT1 axis. Exp Mol Pathol 110: 104283, 2019.
34. Perez Vidakovics MLA, Ure AE, Arrias PN, Romanowski V and Gómez RM: Junin virus induces autophagy in human A549 cells. PLoS One 14: e0218730, 2019.

35. Neubert P, Weichselbaum A, Reitinger C, Schatz V, Schröder A, Ferdinand JR, Simon M, Bär AL, Brochhausen C, Gerlach RG, et al: HIF1A and NFAT5 coordinate $\mathrm{Na}^{+}$-boosted antibacterial defense via enhanced autophagy and autolysosomal targeting. Autophagy 15: 1899-1916, 2019.

36. Nong Q, Li S, Wu Y and Liu D: IncRNA COL1A2-AS1 inhibits the scar fibroblasts proliferation via regulating miR-21/Smad7 pathway. Biochem Biophys Res Commun 495: 319-324, 2018.

37. Wang X, Chu J, Wen CJ, Fu SB, Qian YL, Wo Y, Wang C and Wang DR: Functional characterization of TRAP1-like protein involved in modulating fibrotic processes mediated by TGF- $\beta /$ Smad signaling in hypertrophic scar fibroblasts. Exp Cell Res 332: 202-211, 2015.

38. Pang K, Li B, Tang Z, Yang W, Hao L, Shi Z, Zhang J, Cai L, Li R, Liu Y, et al: Resveratrol inhibits hypertrophic scars formation by activating autophagy via the miR-4654/Rheb axis. Mol Med Rep 22: 3440-3452, 2020 .

39. Shi W, Wu Y and Bian D: p75NTR silencing inhibits proliferation, migration and extracellular matrix deposition of hypertrophic scar fibroblasts by activating autophagy through inhibiting PI3K/Akt/mTOR pathway. Can J Physiol Pharmacol, Jul 29, 2020 (Epub ahead of print). doi: 10.1139/cjpp-2020-0219.

40. De Risi M, Torromino G, Tufano M, Moriceau S, Pignataro A, Rivagorda M, Carrano N, Middei S, Settembre C, AmmassariTeule M, et al: Mechanisms by which autophagy regulates memory capacity in ageing. Aging Cell 19: e13189, 2020.

41. Lanzillotta C,Zuliani I, Vasavda C, Snyder SH,PaulBD, Perluigi M, Di Domenico F and Barone E: BVR-A deficiency leads to autophagy impairment through the dysregulation of AMPK/mTOR axis in the brain-implications for neurodegeneration. Antioxidants (Basel) 9: 671, 2020 .

42. Long J and Pi X: Polyphyllin I promoted melanoma cells autophagy and apoptosis via PI3K/Akt/mTOR signaling pathway. Biomed Res Int 2020: 5149417, 2020.

43. Luo M, Liu Y, Liu N, Shao W, Ming L, Liu J and Xie Y: Proscillaridin A inhibits hepatocellular carcinoma progression through inducing mitochondrial damage and autophagy. Acta Biochim Biophys Sin (Shanghai): Nov 17, 2020 (Epub ahead of print). doi: 10.1093/abbs/gmaa139.

44. Yang J, Wang B, Xu Q, Yang Y, Hou L, Yin K, Guo Q, Hua Y, Zhang L, Li Y, et al: TMEM166 inhibits cell proliferation, migration and invasion in hepatocellular carcinoma via upregulating TP53. Mol Cell Biochem: Nov 16, 2020 (Epub ahead of print). doi: 10.1007/s11010-020-03979-1.

45. Yang Z, Tang Y, Lu H, Shi B, Ye Y, Xu G and Zhao Q: Long non-coding RNA reprogramming (lncRNA-ROR) regulates cell apoptosis and autophagy in chondrocytes. J Cell Biochem 119: 8432-8440, 2018.

46. Urut F, Dede S, Yuksek V, Cetin S, Usta A and Taspinar M: In vitro evaluation of the apoptotic, autophagic, and necrotic molecular pathways of fluoride. Biol Trace Elem Res: Nov 16 , 2020 (Epub ahead of print). doi: 10.1007/s12011-020-02491-3.

(7) $($ ) This work is licensed under a Creative Commons Attribution-NonCommercial-NoDerivatives 4.0 International (CC BY-NC-ND 4.0) License. 